\title{
Zur Frage der Ganzheitsorientierung in der praktischen Wirtschaft, Politik, Pädagogik und in den Wirtschafts- und Sozialwissenschaften - neu gestellt nach dem Lesen der Berthold Beitz-Biographie von Joachim Käppner ${ }^{1}$
}

Zur Frage der Ganzheitsorientierung in der praktischen Wirtschaft, Politik, Pädagogik und in den Wirtschafts- und Sozialwissenschaften - neu gestellt nach dem Lesen der Berthold Beitz-Biographie von Joachim Käppner. ${ }^{2}$

Die hier gemeinte Verwendungsweise des Wortes „Ganzheit“ impliziert, dass es wissenschaftliche Erkenntnis von Individualitäten in ihrer Totalität geben könne, oder aber, dass die Wirklichkeit auf einmal in ihrer Totalität veränderbar sei. Im Unterschied zu den Gestalttheoretikern wird von den Befürwortern ganzheitlicher Kategorien der beschriebenen Art allerdings oftmals die Selektivität und Perspektivität jeder Erkenntnis und jeglichen Verhaltens verkannt. Man sieht dann nicht, dass „,rein sprachliche Darstellungen ... niemals, auch nicht bei der Anwendung noch so sehr zur Erzeugung von Assoziationen geeigneter Gleichnisse und ebenso nicht bei dem Hinweis auf erlebnismäßig noch so eindrucksvolle Zeugnisse, ganz darauf verzichten, den Gegenstand unter Begriffe zu bringen“. ${ }^{3}$ In unserem Sinne ganzheitlich orientierte Praktiker oder Wissenschaftler sind aber eben keineswegs immer und geradezu zwingend spekulativen Bemühungen gefolgt, die in der durch die Aufklärung geprägten Philosophie zu recht auf strikte Ablehnung stießen. ${ }^{4}$

Gerhard Weisser gehört zweifellos zu den bedeutenden ,morphologisch“orientierten Wirtschaftsund Sozialwissenschaftlern, die sich seit den zwanziger Jahren des vorigen Jahrhunderts aus moralisch-politisch-pädagogischen und zugleich betriebswirtschaftlich-sozialökonomischen Gründen um eine von ausschließlicher Rechtsformen-Analyse losgelöste Ganzheits-Betrachtung und Ganzheits-Gestaltung des einzelwirtschaftlichen Bereichs bemüht haben. ${ }^{5}$ Streng genommen haben Autoren wie er - oder in den vergangenen Jahrzehnten etwa auch Frank Schulz-Nieswandt ${ }^{6}$ - dabei natürlich Vorläufer, die im 19. Jahrhundert bis in die Denk- und Handlungsstrukturen Johann Heinrich von Thünens einerseits und im 20. Jahrhundert etwa Walter Rathenaus politisch-praktischen und wissenschaftlichen Arbeiten andererseits reichen.

Im Einzelnen gehören des erstgenannten Autors Bemühungen um eine ausschließlich streng von unten aus aufbauende, also „frei-gemeinnützige“ bzw. „frei-gemeinwirtschaftliche“ Vielfalt von

1 J. Käppner (2010): Berthold Beitz. Die Biographie. Mit einem Vorwort von H. Schmidt, 3. Aufl., Berlin.

2 J. Käppner (2010): Berthold Beitz. Die Biographie. Mit einem Vorwort von H. Schmidt, 3. Aufl., Berlin.

3 G. Weisser (1956): Wirtschaft. In: Handbuch der Soziologie, Stuttgart, S. 975.

4 Siehe dazu zuletzt Die Zeit Nr. 4, 2011, S. 4ff.

5 Vgl. besonders G. Weisser, Form und Wesen der Einzelwirtschaften, Bd. 1, 2. Aufl., Göttingen 1949; Ders., Die Lehre von den gemeinwirtschaftlichen Unternehmen. In: Arch.f.öff.u.fr.U., Bd. 1, Göttingen 1954, S. 3-33.

6 Siehe z. B. dessen weit ausgereifte Studie: Herrschaft und Genossenschaft, Berlin 2003. 
Unternehmenstypen ${ }^{7}$ und des zweitgenannten Schriftstellers etwa das große Werk über einen „Mann ohne Eigenschaften“ dazu. ${ }^{8}$

In der Gegenwart verkörpert Berthold Beitz vielleicht am ehesten ,den am Konsens orientierten Gedanken, welcher der sozialen Marktwirtschaft zugrunde liegt. Und seine Prinzipien, nämlich sozialer Ausgleich und Verantwortung des Unternehmers, die den Verfechtern des schrankenlosen Marktes eben noch als altbacken galten, sind heute in der globalen Krise aktueller denn je“. ${ }^{9}$ Dazu sei auch aus dem Vorwort von Helmut Schmidt zitiert: „,1967 starb Alfried Krupp. Berthold Beitz war Testamentsvollstrecker. Kurz vor seinem Tode hat Alfried Krupp von dem `dem Gemeinwohl verpflichteten Tradition des Hauses' gesprochen. Sie mache es zur Pflicht ,... erwerbswirtschaftliche Überlegungen - so wichtig sie auch sind - nie isoliert zu sehen vom Gebot der Sozialverpflichtung des persönlichen Eigentums“.

Alfried Krupp habe sich zu recht ganz und gar auf Berthold Beitz verlassen. „1968 ging die 'Friedrich Krupp $\mathrm{GmbH}^{\prime}$ in den Besitz der gemeinnützigen Stiftung über... Der Krupp- Stiftung ist durch ihre Satzung geboten, die Einheit des Unternehmens zu wahren und dessen Erträge gemeinnützigen Zwecken zuzuführen. Um die doppelte Verpflichtung hat Bertold Beitz immer wieder ringen müssen. Letztlich ist ihm - trotz der unaufhaltsamen Fusionen der deutschen Stahlindustrie - dieser Spagat bis heute gelungen... Die Erfüllung moralischer Pflicht hat Vorrang vor der Mehrung des eigenen Wohlstandes. Moral ergibt sich nicht aus dem Wettbewerb. Die KruppStiftung ist heute Großaktionär von Thyssen-Krupp. Sie hat im Laufe von 43 Jahren unter Berthold Beitz' Leitung 600 Millionen Euro für gemeinnützige Zwecke ausgeschüttet... zugunsten unserer Gesellschaft und Kultur.... ${ }^{10}$

Die detaillierten biographischen Ausführungen in seiner excellenten Biographie führen den Autor selbst schließlich zu folgender These über den ganzheitlich orientierten Zustand des ThyssenKrupp-Konzerns vor der großen Wirtschafts- und Finanzkrise des Jahres 2009: „Vorstand und Betriebsrat finden die wärmsten Worte füreinander, das für einen Großkonzern mit mehr als 200 000 Arbeitsplätzen in der Tat nicht selbstverständlich ist.“ Im „schönsten Neudeutsch“ bezeichne der Betriebsrat seine Haltung als ,,comanagement-orientiert“: ,,man ist stolz auf das Erreichte und die starke Position der Arbeitnehmer. Beitz gilt ihnen dabei als Symbol einer in den Jahren ökonomischer Gier anderswo vergessenen oder belächelten Sozialpartnerschaft. 'Er hat den Anstand und Moral - und behandelt uns auf gleicher Augenhöhe', sagt Schlenz. Die Gewerkschaften fühlen sich durch ihn als Gleichwertige behandelt, als Partner, die ja auch nur legitime Interessen vertreten. Das sind Dinge, die in der globalisierten Wirtschaft leider immer mehr verloren gehen'. In der Tat ist es erstaunlich: „In dem Konzern, der ja immerhin drei Traditionsunternehmen vereient, beginnt sich eine corporate identity zu entwickeln, die Züge des alten Krupp'schen Wir-Gefühls trägt“. ${ }^{11}$ „,Ohnehin wir die Stiftung nach Beitz eine andere sein. Die Macht über sie, über das Erbe

7 Siehe dazu W. W. Engelhardt (2008): Johann Heinrich von Thünen als Vordenker einer Sozialen Marktwirtschaft, Marburg. Zuletzt siehe vom gleichen Verfasser (2010): Renaissance der Frei-Gemeinwirtschaftlichkeit und der Vielfalt der Unternehmenstypen. Die neue Aktualität von Johann Heinrich von Thünen. In: ZögU, 33. Jg., Heft 2, S. 159-167.

8 Vgl. dazu im künstlerischen Bereich bis heute unübertroffen R. Musli (1952): Der Mann ohne Eigenschaften. Roman, Hamburg.

9 So J. Käppner, Zur Einführung. In: Ders., Berthold Beitz, a.a.O., S. 20.

10 H. Schmidt, Vorwort. In: J. Kräppner, Berthold Beitz, a.a.O., S. 13.

11 J. Käppner: Berthold Beitz, a.a.O., S. 512. Thomas Schlenz war zu diesem Zeitpunkt der Betriebsratschef. 
Krupps, ist Besitz von Alfried Krupp faktisch auf Lebenszeit übertragen worden, aber eben nur ihm. Sein Nachfolger wird mit anderen Strukturen leben müssen, er wird sich Wahlen stellen müssen in einer Stiftung, die sein wird wie andere auch und eben kein auf Beitz zugeschnittenes Imperium mehr". ${ }^{12}$

In Ausführungen Käppners über das „Vermächtnis“ von Beitz und in Bemerkungen über ein „Nachwort“ zu seiner großen Biographie heißt es zur Frage: „Was hält die Gesellschaft noch zusammen? Diese Frage stellen heute, nach dem Ende der Ideologien und in der globalen Krise nicht mehr nur Soziologen und evangelische Akademieseminare; sie ist zu einer Grundsatzfrage in einer Welt geworden, die sich rasch wandelt.“ Beitz hatte ,an sich den Anspruch, mit offenen Visier zu fechten und im Sinne einer Verantwortlichkeit für das Ganze, und er tat es, wenn er es im Interesse des Ganzen für geboten hielt. Doch er hat nicht vergessen, dass er selbst von sehr weit unten kam.“ „'Es ist wichtig, dass Menschen ihren Kindern die Möglichkeit bieten können, aufzusteigen und weiter nach oben zu können. Und dazu gehört, dass man sie nicht von oben herab behandelt'. Es ist dies, in sehr persönlichen Worten, eine Art Vermächtnis““. ${ }^{13}$

„Es gibt einen Ausdruck im Grundgesetz, der die Beitz'sche Philosophie auf den Punkt bringt. 'Eigentum verpflichtet', heißt es in Art. 14 Abs. 2. 'Sein Gebrauch soll zugleich dem Wohle der Allgemeinheit dienen'." Nach dem Urteil von Beitz hatten auch im nationalsozialistischen Staat die Menschen ,eine Wahl, und sie wählten falsch.“ Er selbst stand im Kriege ebenfalls vor einer Wahl. „Er entschied sich dafür, den Verfolgten zu helfen, er riskierte sein Leben für das, was er für richtig erachtete.“”,In Polen, aber auch später, als Wegbereiter der Aussöhnung mit dem Osten und mit den Opfern der Nazidiktatur, als Unternehmer, der heute wieder als Vorbild wie aus der lange schon als fast versunkenen geltenden Zeit der sozialen Marktwirtschaft gilt." Die zweifellos am Gesamtwohl orientierte Persönlichkeit namens Beitz zitiert heute gern einen Satz, der dem größten Staatsmann des klassischen Athens, Perikles, zugeschrieben wird: „Das Geheimnis des Glücks ist die Freiheit, und das Geheimnis der Freiheit ist der Mut.“ „Auf Berthold Beitz passt dieser Satz genau. Sein Lebensweg zeigt, was ein Mensch erreichen kann, der die Freiheit des Handelns mit Mut und Verantwortung nutzt. Und wie er trotz aller Mühen ein glücklicher Mensch werden kann. Doch, einzelne Menschen können die Welt verändern. Jedenfalls ein Stück weit". 14

12 J. Käppner: Berthold Beitz, a.a.O., S. 518.

13 J. Käppner: Berthold Beitz, a.a.O., S $524 \mathrm{ff}$.

14 J. Käppner: Berthold Beitz, a.a.O., S. 525ff. 
Ganzheitsorientierung in Wirtschaft, Politik, Pädagogik und Wirtschafts- und Sozialwissenschaften

\section{Literaturverzeichnis}

Engelhardt, Werner W. (2008), Johann Heinrich von Thünen als Vordenker einer Sozialen Marktwirtschaft, Marburg. Engelhardt, Werner W. (2010), Renaissance der Frei-Gemeinwirtschaftlichkeit und der Vielfalt der Unternehmenstypen. Die neue Aktualität von Johann Heinrich von Thünen, in: ZögU, 33. Jg., Heft 2, S. 159-167.

Käppner, Joachim (2010), Berthold Beitz. Die Biographie. Mit einem Vorwort von Helmut Schmidt, Berlin.

Musil, Robert (1952), Der Mann ohne Eigenschaften. Roman, Hamburg.

Schmidt, Helmut (2010), Vorwort, in: Joachim Kräppner, Berthold Beitz. Die Biographie, Berlin, S. $11 \mathrm{ff}$.

Thumann, Michael (2011), Der tunesische Funke. Knüppel und Geschenke: Wie arabische Herrscher jetzt gegen Oppositionelle vorgehen, in: Die Zeit, Nr. 4, S. 4ff.

Weisser, Gerhard (1949), Form und Wesen der Einzelwirtschaften, Bd. 1, 2. Aufl., Göttingen.

Weisser, Gerhard (1954), Die Lehre von den gemeinwirtschaftlichen Unternehmen, in: Arch.f.öff.u.fr.U., Bd. 1, Göttingen.

Weisser, Gerhard (1956), Wirtschaft, in: Werner Ziegenfuß (Hrsg.), Handbuch der Soziologie, Stuttgart. 